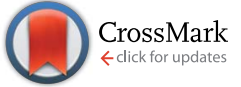

Cite this: RSC Adv., 2017, 7, 12745

Received 3rd January 2017

Accepted 13th February 2017

DOI: $10.1039 / c 7 r a 00078 b$

rsc.li/rsc-advances

\section{Value-additive utilization of agro-biomass: preparation of cellulose triacetate directly from rice straw as well as other cellulosic materials $\uparrow$}

\author{
Amita Sharma,,$^{\mathrm{a}}$ Santosh Kumar Giri,,$^{\mathrm{b}}$ K. P. Ravindranathan Kartha*b \\ and Rajender S. Sangwan*a
}

Non-forage crop residues like rice straw are among the most important and pertinent renewable biomass available for utilization for producing biofuels, specialty chemicals, aromatics etc. Recovery of cellulose from them and its processing for products are two key steps of value addition to these materials that are available in abundance. Of these, rice straw is perhaps the most recalcitrant of all. In this study, production of cellulose triacetate (CTA) from cellulose by three different (alternate) practical methods are reported. It has been demonstrated that cellulose in the form of microcrystalline cellulose (MCC), other celluloses commercially available from chemical suppliers, cotton linters, and rice straw as such (directly after powdering) are suitable as substrates for the reaction. The methods make use of either acetic acid, acetyl chloride or isopropenyl acetate (IPA) as the acetyl donor in the presence of methane sulfonic acid (a cheap and green reagent) as the acid catalyst. The conventional acetyl donor agent, acetic anhydride is also a suitable alternative reagent for the purpose. The post-reaction work-up procedure involves neutralization and filtration as the only two simple successive steps. The processes reported thus eliminate the need for using restricted/toxic chemicals (acetic anhydride and pyridine, respectively) and provide cellulose tri-O-acetate of high purity in excellent yields. Preparation of CTA directly from rice straw has been achieved in $>70 \%$ yield, representing acetylation of the straw cellulose into its triacetate to an extent of $90 \%$. With IPA as the acyl donor, acetone was a valuable and recoverable co-product. Although not demonstrated in this study, the residue from the acetylation reaction can be utilized for the recovery of value-added materials such as fine silica.

\section{Introduction}

Carbohydrates, namely, cellulose, hemicelluloses, starch and sucrose together constitute approximately $75 \%$ of plant biomass in which cellulose forms the major component amounting to more than $40 \%$ of the total biomass composition., ${ }^{1,2}$ Although cellulose is organic and biodegradable, is nontoxic and is built of anhydroglucose units, its unique macromolecular structure makes it insoluble in water as well as organic solvents. The fact that it undergoes decomposition before melting also makes it less amenable to industrial processing. To a great extent this problem is usually addressed by its conversion to soluble derivatives. Derivatized cellulosics find

${ }^{a}$ Center of Innovative and Applied Bioprocessing (CIAB), C-127, Phase-8, S.A.S. Nagar, Mohali-160071, Punjab, India. E-mail: sangwan@ciab.res.in; Tel: +91-172-2292028 ${ }^{b}$ Department of Medicinal Chemistry, National Institute of Pharmaceutical Education and Research, S.A.S Nagar, Punjab-160062, India. E-mail: rkartha@niper.ac.in; Fax: +91-172-2214692; Tel: +91-172-2292028

$\dagger$ Electronic supplementary information (ESI) available. See DOI: 10.1039/c7ra00078b

\$ Amita Sharma and Santosh Kumar Giri are co-first co-authors who contributed equally. many important applications in the fiber, paper, optical film, membrane, coating, and paint industries. ${ }^{3}$ Among the important cellulose derivatives are the esters (acetate, sulfonate, etc.) and the ethers (alkyl, hydroxyalkyl, carboxyalkyl, etc. $)^{4}$ that serve well in the textile and various polymer industries..$^{5-9}$ Cellulose triacetate (CTA) is one of the oldest man made cellulose esters that have been used widely in the industry and is generally prepared by treating cellulose with acetic anhydride in acetic acid in the presence of $\mathrm{H}_{2} \mathrm{SO}_{4} / \mathrm{HClO}_{4}$ as catalyst; and often suffers from the limitations of low degree of substitution (DS) and poor yields for various reasons. ${ }^{10}$ Use of $\mathrm{H}_{2} \mathrm{SO}_{4}$ often also leads to residual sulfur (from sulfate ester substitution) in the final product. Liebert and Heinze reported that treatment of cellulose with vinyl acetate in DMSO/TBAF leads to the formation of CTA but is accompanied by side reactions such as oxidation. ${ }^{11}$ Homogeneous reaction conditions for the purpose of smooth reactions are also reportedly achieved by using various ionic liquids ${ }^{\mathbf{1 2 , 1 3}}$ such as 1-allyl-3-methylimidazolium chloride, ${ }^{14}$ 1-butyl-3-methylimidazolium chloride, ${ }^{15,16}$ those based on choline chloride and $\mathrm{ZnCl}_{2}$, as in $[\mathrm{ChCl}]\left[\mathrm{ZnCl}_{2}\right]_{2}{ }^{17}$ etc. Moreover, for the synthesis of CTA the $\mathrm{Ac}_{2} \mathrm{O}-\mathrm{I}_{2}$ system originally proposed for simple sugars ${ }^{\mathbf{1 8}}$ has also been reported either at 
$100{ }^{\circ} \mathrm{C}$ under mechanical stirring ${ }^{19}$ or under a microwave. ${ }^{\mathbf{2 0 , 2 1}}$ Biswas and associates ${ }^{22}$ have also reported an acetic anhydridebased process of obtaining CTA directly from rice hull (i.e. rice grain husk) but the yields were far lower than those with isolated cellulose from the feedstock. Also, rice husk is a minor (rice grain to husk ratio of only $1: 0.2$ ) by-product most of which is being profitably used in the milling industry itself for boiler operations and direct power generation. It is in this context that we like to now report our findings that CTA can be conveniently prepared by reacting cellulose [in the form of microcrystalline cellulose (MCC), cellulose or rice straw] with an acyl donor reagent such as isopropenyl acetate or acetyl chloride or glacial acetic acid in the presence of methanesulfonic acid $\left(\mathrm{MeSO}_{3} \mathrm{H}\right.$, MeSA). ${ }^{23}$ The method does not require use of $\mathrm{Ac}_{2} \mathrm{O}$, the acetyl donor whose supply is restricted by law, and is simple, economical, and easy to operate and green. The reaction produces no toxic waste and is suitable for scale up. On using IPA as the acyl donor, the by-product, namely, acetone formed can be collected fully by simple distillation.

\section{Results and discussion}

We recently had noted that MeSA is an excellent green catalyst for the acetylation of hydroxyl and sulfhydryl group-containing substrates such as alcohols and thiols. This prompted us to investigate its applicability in the acetylation of polymeric and difficult-to-acetylate carbohydrates such as cellulose. The much investigated and most widely used acetyl donor, $\mathrm{Ac}_{2} \mathrm{O}$ itself was chosen as the reagent of choice for the initial experiments employing MCC as the substrate for acetylation; and this must also serve as control for comparison of the results of the experiments to follow. Thus, when MCC ( $1 \mathrm{~g})$ was treated with $\mathrm{Ac}_{2} \mathrm{O}(10 \mathrm{~mL})$ in the presence of $\mathrm{MeSA}(1 \mathrm{~mL})$ for $24 \mathrm{~h}$ at $42{ }^{\circ} \mathrm{C}$, a near-colourless solid product $(1.65 \mathrm{~g}, 93 \%$ based on the weight expected for CTA; entry 1, Table 1) was indeed obtained. The overall reaction can be depicted as shown in Scheme 1. The product was freely soluble in organic solvents such as EtOAc, $\mathrm{CH}_{2} \mathrm{Cl}_{2}, \mathrm{CHCl}_{3}$, etc. and, on removal of the solvent by evaporation, it formed a transparent and clear film indicating it to be the acetate. The acetylated product thus obtained when analysed by IR did not show any hydroxyl absorption bands in its IR spectrum (data not shown) and the signals observed in the ${ }^{1} \mathrm{H}$ NMR spectra (see p. S2 of the ESI $\dagger$ ) were in accordance with what was expected for a per- $O$-acetylated $(1,4)$ - $\beta$-linked glucan, confirming its identity as the tri- $O$-acetate from MCC. Moreover, the product did not show presence of sulfur when subjected to elemental analysis for sulfur. It may also be pointed out that although $\mathrm{MeSA}-\mathrm{Ac}_{2} \mathrm{O}$ system can arguably be considered capable of doing acetolytic cleavage of interglycosidic bonds in carbohydrates, their stability to acetolysis in $\mathrm{MeSA}-\mathrm{Ac}_{2} \mathrm{O}$ system

Table 1 Optimization of reaction conditions for the preparation of CTA using various cellulosic substrates and its application for the preparation of CTA directly from rice straw ${ }^{a}$
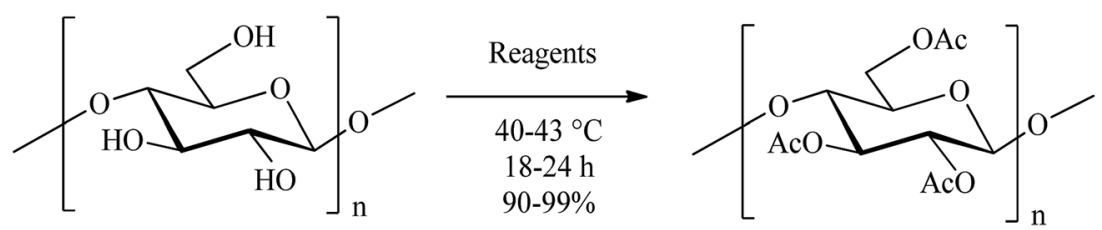

\begin{tabular}{|c|c|c|c|c|c|c|}
\hline \multirow{2}{*}{$\begin{array}{l}\text { Entry } \\
1\end{array}$} & \multirow{2}{*}{$\begin{array}{l}\text { Substrate } \\
\text { MCC }\end{array}$} & \multicolumn{3}{|c|}{ Reagents (mL) } & \multirow{2}{*}{$\frac{\text { Reaction }^{b} \text { time }(\mathrm{h})}{24}$} & \multirow{2}{*}{$\begin{array}{l}\text { Yield }^{c}(\%) \\
93\end{array}$} \\
\hline & & $\mathrm{Ac}_{2} \mathrm{O}(10)$ & - & $\operatorname{MeSA}(1)$ & & \\
\hline 2 & & IPA (10) & - & MeSA (1) & 48 & 6 \\
\hline 4 & & IPA (10) & AcOH (10) & $\operatorname{MeSA}(1)$ & 18 & 99 \\
\hline 5 & & IPA (10) & $\mathrm{AcOH}(10)$ & $\operatorname{MeSA}(0.5)$ & 20 & 95 \\
\hline 6 & & AcOH (20) & - & $\operatorname{MeSA}(1)$ & 18 & 98 \\
\hline 9 & & $\mathrm{AcOH}(20)$ & $\operatorname{AcCl}(5)$ & - & 18 & 90 \\
\hline 10 & & $\mathrm{AcOH}(20)$ & $\operatorname{AcCl}(2.5)$ & - & 18 & 85 \\
\hline 11 & & AcOH (10) & $\operatorname{AcCl}(2.5)$ & - & 18 & 80 \\
\hline 12 & Cellulose & IPA (10) & $\mathrm{AcOH}(10)$ & $\operatorname{MeSA}(1)$ & 18 & 98 \\
\hline 13 & & $\mathrm{AcOH}(10)$ & - & MeSA (1) & 18 & 60 \\
\hline 14 & & $\mathrm{AcOH}(20)$ & $\operatorname{AcCl}(5)$ & - & 18 & 55 \\
\hline 19 & & $\mathrm{Ac}_{2} \mathrm{O}(10)$ & $\mathrm{AcOH}(10)$ & MeSA (1) & 24 & $35^{d}$ \\
\hline 20 & & $\mathrm{Ac}_{2} \mathrm{O}(20)$ & - & MeSA (1) & 24 & $40^{d}$ \\
\hline
\end{tabular}

${ }^{a}$ Substrate $(1 \mathrm{~g})$ was treated with reagents at $42{ }^{\circ} \mathrm{C} .{ }^{b}$ Each experiment was repeated twice for checking the reproducibility. ${ }^{c}$ Isolated yield is reported in all the cases. ${ }^{d}$ Based on the percent weight gain in the estimated cellulose content of rice straw. 


$$
\begin{gathered}
\mathrm{MeSO}_{3} \mathrm{H} \\
\text { Cellulose }-(\mathrm{OH})_{3}+\mathrm{Ac}_{2} \mathrm{O} \stackrel{(\mathrm{H})}{\longrightarrow} \text { Cellulose }-(\mathrm{OAc})_{3}+\mathrm{AcOH}
\end{gathered}
$$

Scheme 1 MeSA-catalysed acetylation of cellulose using $\mathrm{Ac}_{2} \mathrm{O}$ as the acyl donor.

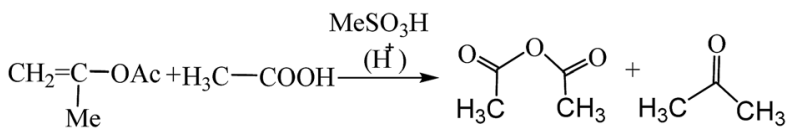

Scheme 2 MeSA-catalysed reaction of IPA with $\mathrm{AcOH}$.

in the case of di- and trisaccharides as well as $\beta$-cyclodextrin has already been demonstrated recently.

A possible limitation of this method, as is the case with all those that necessitate the use of $\mathrm{Ac}_{2} \mathrm{O}$, however, is that it involved use of a substance $\left(\mathrm{Ac}_{2} \mathrm{O}\right)$ that is a somewhat restricted chemical commodity. As an alternative, therefore, the use of isopropenyl acetate (IPA) as the acetylating agent was explored simultaneously (Table 1). Acetylation of a variety of hydroxy compounds (alcohols, enols and phenols) as well as amines using IPA has been described in a paten $t^{24}$ and was followed by detailed publications of the work subsequently by Hull and Hagemeyer ${ }^{25}$ as well as others; $;^{26-30}$ and the catalysts employed include sulfuric acid and $p$-toluene sulfonic acid..$^{25}$ The reactions were found to proceed smoothly and resulted in very high yields of the acetylated product under neat conditions and in the case of some of the amines, it was obtained rather spontaneously (without the aid of an added catalyst). Thus, the process was proved to be useful for the chemoselective acetylation of an amino group in hydroxyalkyl-substituted amino compounds, as for example, in the preparation of $\mathrm{N}$-(2-hydroxyethyl)-acetamide from monoethanolamine. A prominent feature of this work ${ }^{25}$ in the context of green chemistry is that the desired acetate/acetamide and the by-product of the reaction (acetone) could be separated efficiently by distillation, making good use of the differences in their boiling points. A similar work on the acetylation of simple alcohols and amines using IPA was again reported recently by Pelagalli and coworkers. ${ }^{31}$ In view of the findings by Hull and co-workers ${ }^{24}$ for simple alcohols and amines therefore, when MCC $(1 \mathrm{~g})$ was treated with IPA (neat, $10-20 \mathrm{~mL}$ ) in the presence of MeSA $(1 \mathrm{~mL})$ for up to 3 days, CTA could be isolated after an aqueous work up and filtration in a yield of up to $51 \%$ (entries 2 and 3, Table 1). In the absence of the acidic catalyst no product was obtained (results not listed in the table). Nevertheless, the above results were not satisfactory so far as the product yield and the reaction time were concerned. It may be recalled ${ }^{26,27}$ that the reaction of IPA with $\mathrm{AcOH}$ in the presence of strong acids can generate $\mathrm{Ac}_{2} \mathrm{O}$ in situ (along with the by-product, acetone) as depicted in Scheme 2. Further, in view of the fact that $\mathrm{AcOH}$ can serve as an excellent solvent for CTA, the foregoing reaction of cellulose with IPA was also tried in the presence of added $\mathrm{AcOH}$ (entry 4, Table 1). Quite satisfyingly, under these conditions CTA could be isolated in near-quantitative yields (entry 4, Table 1 ) after $18 \mathrm{~h}$ of stirring. Reducing the catalyst concentration to half the above was found to marginally affect the CTA yields ( $95 \%$ yield, entry 5 , Table 1). The NMR spectra obtained for the CTA prepared from MCC by the MeSA-IPA-AcOH method as discussed above (Fig. 1 and 2) clearly demonstrated that the product obtained was exclusively the triacetate (CTA). The absence of any hydroxyl absorption signal in the IR spectra and the absence of sulfur on elemental analyses, etc. were also in accordance with the expected results.

Further, in the light of our earlier finding that MeSA was capable of transferring the acyl group from $\mathrm{AcOH}$ to simple alcohols to yield the corresponding acetate esters, ${ }^{23}$ this reaction was also recruited to test its feasibility for the acetylation of macromolecules like cellulose. When the reaction of MCC was carried out in neat AcOH (without any added IPA) in the presence of MeSA, the desired product CTA could be isolated in similar yields ( $98 \%$, entry 6 , Table 1 ) with reaction proceeding as per Scheme 3 shown below.

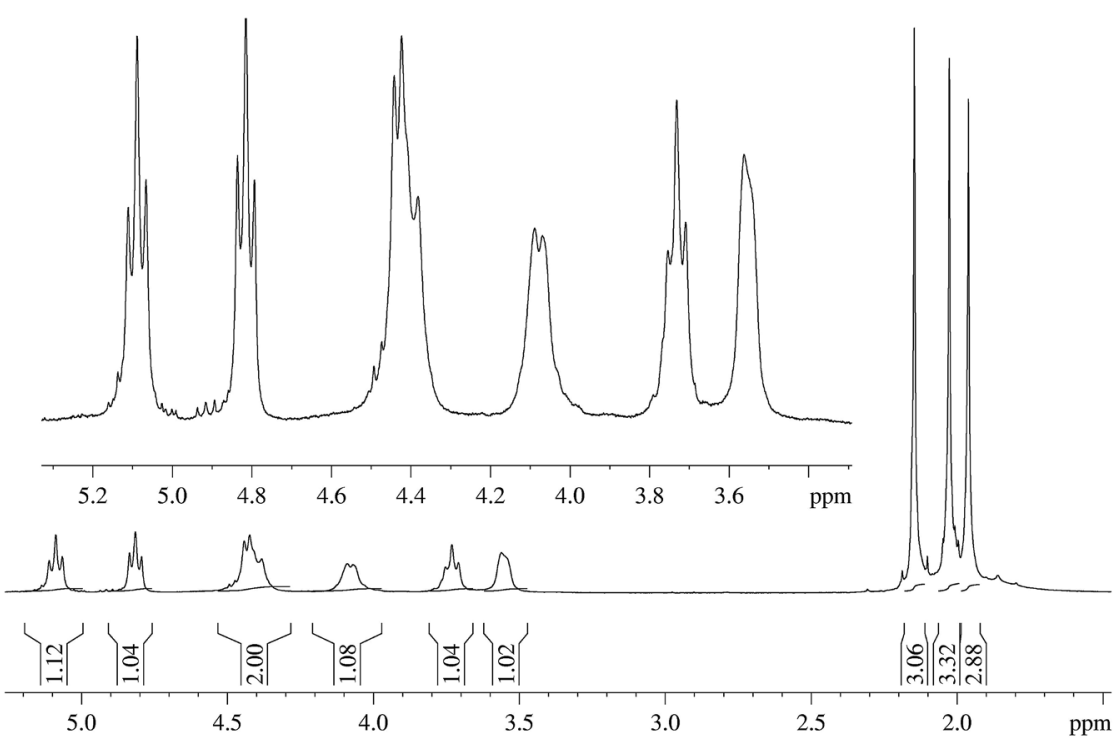

Fig. $1{ }^{1} \mathrm{H}$ NMR spectrum of CTA prepared from MCC by the MeSA-IPA-AcOH method and the expanded 3.5-5.2 ppm region of the spectrum. 


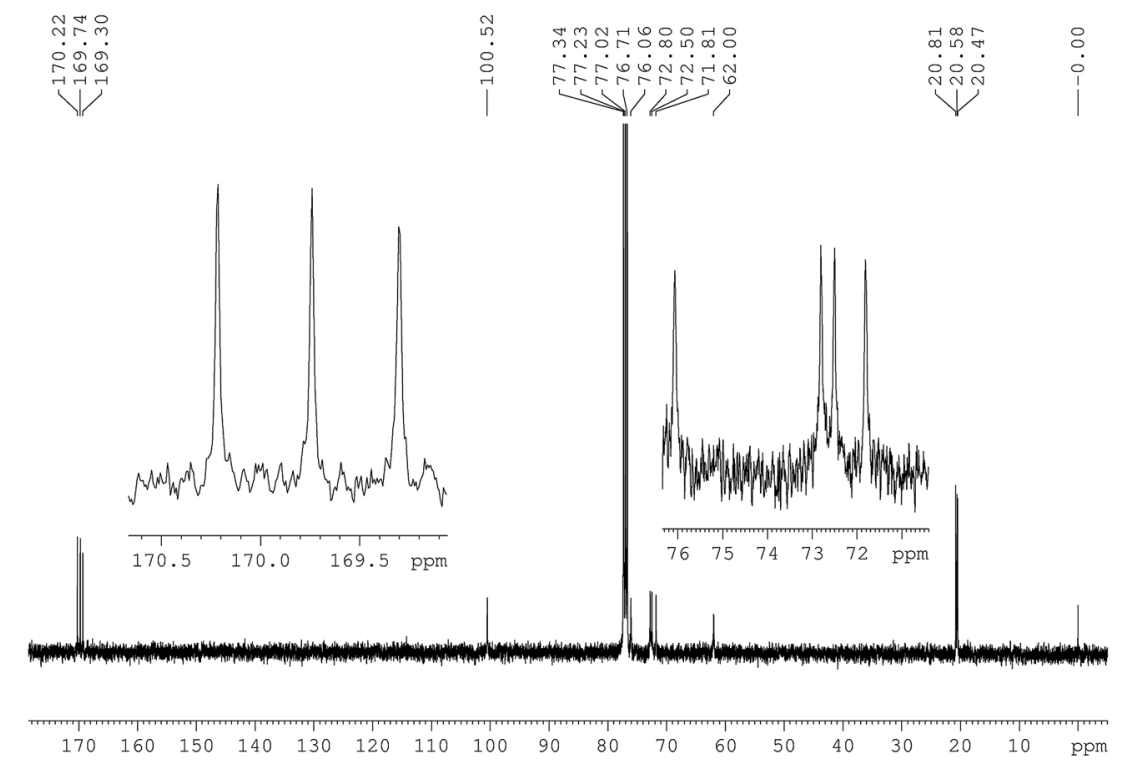

Fig. $2{ }^{13} \mathrm{C}$ NMR spectra of the triacetate (CTA) prepared from MCC using MeSA-IPA-AcOH and the region 70-76 ppm and the region $169-$ 170.5 ppm expanded.

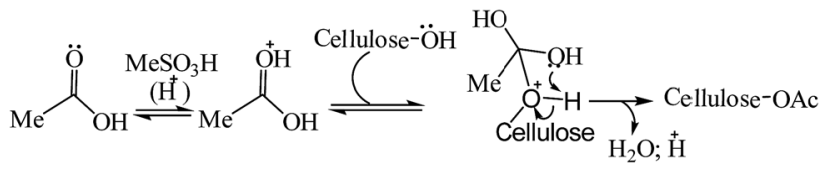

Scheme 3 MeSA-catalysed reaction of cellulose with $\mathrm{AcOH}$.

Here again, reducing the quantity of $\mathrm{AcOH}$ employed for the reaction to half significantly affected the yield of CTA ( $42 \%$ only, entry 7, Table 1); and when this was also accompanied by the use of a reduced quantity of the catalyst (MeSA) in the reaction mixture, it led to further depletion of yields of CTA (entry 8, Table 1). Thus, it was observed that for a quantitative conversion of the polysaccharide substrate in gram-scale reactions, use of approximately $20 \mathrm{~mL} \mathrm{AcOH}$ per gram MCC was best suited.

On the basis of the argument that reaction of $\mathrm{AcCl}$ with $\mathrm{AcOH}$ can also generate $\mathrm{Ac}_{2} \mathrm{O}$ in situ (eqn (1) below), a set of reactions employing a mixture of $\mathrm{AcOH}$ and $\mathrm{AcCl}$ was also performed (entries 9-11, Table 1). The results clearly showed that excellent yields (up to 90\%) of CTA can be obtained using $\mathrm{AcOH}: \mathrm{AcCl}$ in a ratio of $4: 1$ with $20 \mathrm{~mL} \mathrm{AcOH} / \mathrm{g}$ MCC. It can be seen from Table 1 that yields were reduced to $80-85 \%$ on reducing the quantum of $\mathrm{AcCl}$ or $\mathrm{AcOH}$ used for the reaction (entries 10 and 11, Table 1).

$$
\mathrm{AcOH}+\mathrm{AcCl} \rightleftharpoons \mathrm{Ac}_{2} \mathrm{O}+\mathrm{HCl}
$$

$\mathrm{HCl}$, the by-product formed in the reaction, served as an in situ catalyst for the acyl transfer reaction. This conclusion was forthcoming from the fact that presence of both $\mathrm{AcOH}$ and $\mathrm{AcCl}$ was essential for the smooth and significant progress of the reaction. Experiments conducted by exclusion of either of them did not produce the desired result, even when the reaction was allowed to go up to 4 days, (data not shown in the table). Thus, a combination of $\mathrm{AcOH}-\mathrm{AcCl}$ also formed an excellent system for efficient acetylation of MCC. However, the fact that AcCl is a corrosive reagent and fuming in air by nature, could prove to be practical limitations of this method in large scale preparations unless addressed by adopting the appropriate reaction kettles and methods of handling such reagents. An overlay of the ${ }^{1} \mathrm{H}$ NMR spectra of the triacetates prepared from MCC by the four different methods enumerated above have been presented in Fig. 3 for enabling a direct comparison of these methods in a facile manner. In all the cases, it can be seen that, the triacetate (CTA) has unambiguously been the reaction product obtained. After optimization of the above methods for acetylation using MCC as the model cellulosic substrate, the three nonacetic anhydride acetylation systems (IPA-AcOH-MeSA, AcOH-MeSA and $\mathrm{AcOH}-\mathrm{AcCl}$, methods A, B and C, respectively) were separately evaluated against the commercially available cellulose (entries 12-14, Table 1). It was found (i) that the commercially available cellulose could be acetylated highly efficiently and with ease under these conditions affording the ester as a tri-O-acetate (see Fig. 3 for the NMR spectra) in all the three cases and, (ii) that the best results in terms of the yield of CTA were obtained by the IPA-AcOH-MeSA method.

Now, it may be recalled that most of the methods hitherto reported in the literature for the preparation of CTA from crude cellulosics such as straw from a crop, involved a priori alkali extraction step to retrieve cellulose from them. The treatment involves use of a strong alkali such as $\mathrm{NaOH}$; and is followed by the acetylation step by the conventional $\mathrm{Ac}_{2} \mathrm{O}$-method. Therefore it could not have perhaps been surprising that when rice grain hull/husk was employed by Biswas and co-workers (2006) directly for acetylation using the $\mathrm{Ac}_{2} \mathrm{O}-\mathrm{H}_{2} \mathrm{SO}_{4}$ route, the yields obtained (13.5\%) were significantly poorer than those obtained $(25 \%)$ from cellulose isolated from the biomass. In view of the 


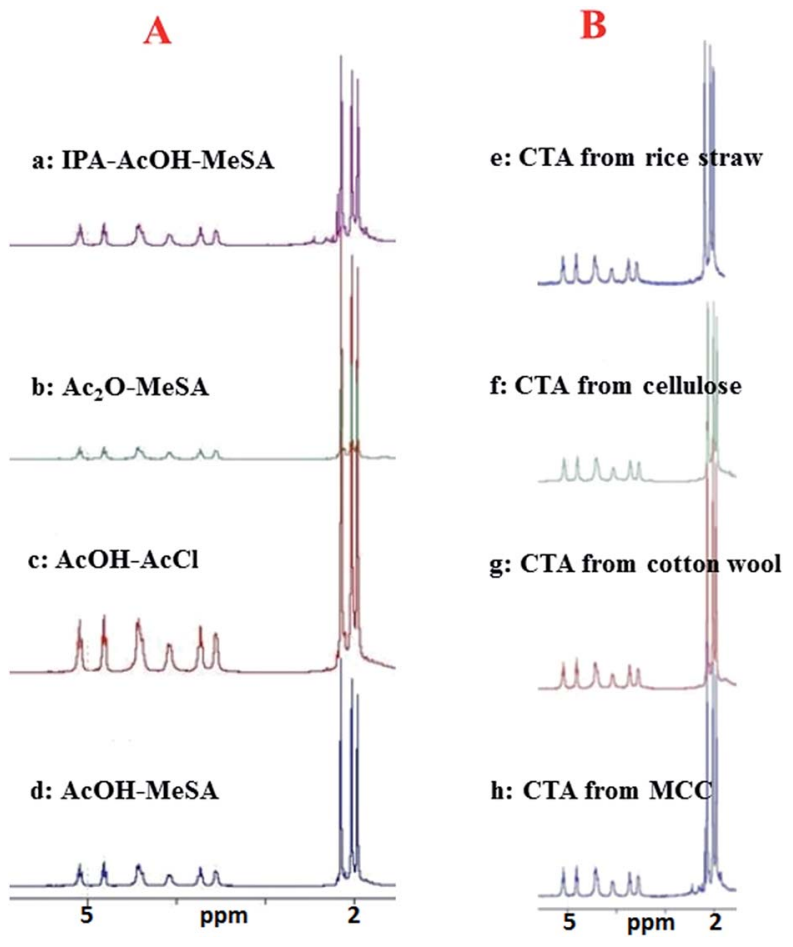

Fig. 3 Comparison of ${ }^{1} \mathrm{H}$ NMR spectra of different CTA preparations. Column A: spectra of CTA prepared from MCC using the four different methods of acetylation reported (entries a-d). Column B: CTA prepared from four different cellulosic materials (including rice straw $\&$ cotton linters, entries (e-h)) using IPA-AcOH-MeSA-method.

above situation, an investigation on the applicability of the above described methods for the preparation of CTA directly from rice straw was felt important. Thus, rice straw obtained from a local agricultural field was pulverized in a mechanical mill and the powder was subjected to acetylation by the different methods described above (entries 15-20, Table 1). Indeed, when the methods were extended to the pulverized rice straw (Fig. 4a) as the substrate, CTA could be obtained in very impressive yields (based on an estimated $40 \%$ cellulose content) directly after the workup as noted above; and the methods $A$ and B (IPA-AcOH-MeSA and AcOH-MeSA, respectively) were proved best with respect to the yields obtained. The ${ }^{1} \mathrm{H}$ NMR spectrum of the crude CTA thus obtained (Fig. 5a) showed the product to be the desired tri-O-acetate (CTA) along with some residual contaminants originating from the crude straw used as the

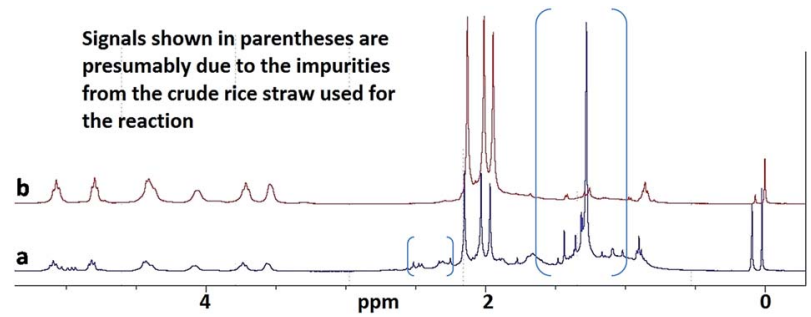

Fig. $5{ }^{1} \mathrm{H}$ NMR spectra of CTA obtained by the direct acetylation of rice straw: (a) the crude product straight after the aqueous workup of the reaction mixture; (b) after removing the $\mathrm{CHCl}_{3}$ insolubles.

substrate for the reaction. It may be recalled that rice straw contains substantial quantities of silica; and silica-OH protons show signals around the $2 \mathrm{ppm}$ region in the solid state ${ }^{1} \mathrm{H}$ $\mathrm{NMR}^{32-34}$ as obtained in the high field region of the spectrum shown in Fig. 5b. Moreover, the product could be made virtually free from these contaminating materials by a simple dissolution of the crude CTA preparation in $\mathrm{CH}_{2} \mathrm{Cl}_{2}$ or $\mathrm{CHCl}_{3}$ (whereupon the contaminants coalesced into a solid sediment) followed by filtration. This was evidenced by the new ${ }^{1} \mathrm{H}$ NMR spectrum (reproduced in Fig. 5a) of the CTA thus obtained by the dissolution technique.

Moreover, the X-ray diffractograms of the triacetate obtained before and after the removal of the chloroform-insolubles show clearly the elimination of the peak at a $2 \theta$ of $22.14^{\circ}$ typical for the silica (Fig. 6, see also p. S3 of the ESI†) in the latter. Photomicrographs of films of crude CTA preparations obtained (by the evaporation of their respective solutions in $\mathrm{CH}_{2} \mathrm{Cl}_{2}$ ) from rice straw (directly) and from the MCC can be seen in Fig. 4 (Fig. $4 \mathrm{c}$ and b, respectively). Likewise the SEM images of the CTA preparations described above revealing their high degree of comparability in terms of their fine structural features can also be confirmed (see p. S4 of the ESI $\dagger$ ). It may also be emphasized that the $\mathrm{CH}_{2} \mathrm{Cl}_{2} / \mathrm{CHCl}_{3}$ insolubles mentioned above are in the process of being subjected to investigations for exploring the possibilities for recovering the rice straw silica possessing great market potential.

A brief review of the methods for the synthesis of CTA from cellulose published in the literature in the past years was felt useful in this context for their due comparison with the methods developed in the current investigation as described above. Table 2 summarises some of the noted methods used for

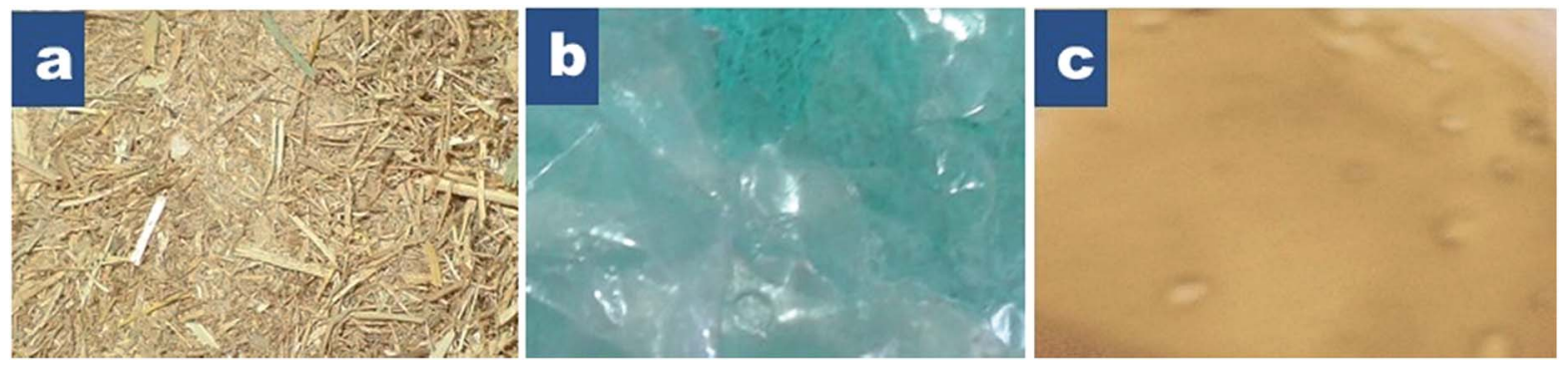

Fig. 4 Photomicrographs of (a) rice straw, (b) CTA obtained from MCC by method A, and (c) CTA prepared directly from rice straw by method A. 


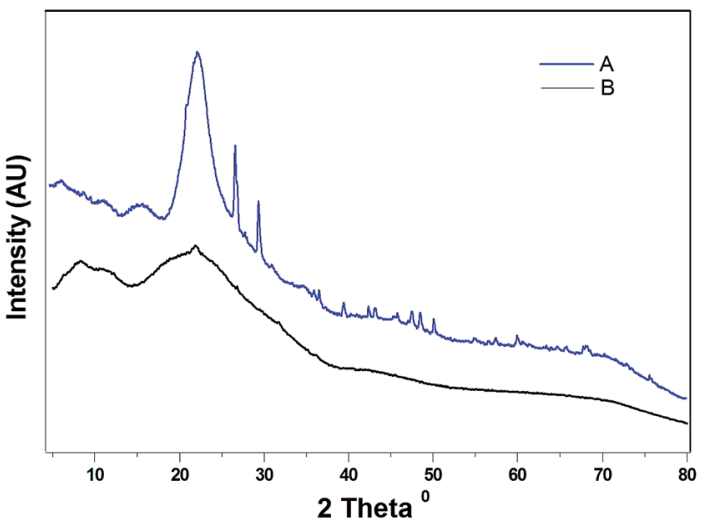

Fig. 6 X-ray diffractograms of CTA obtained by the direct acetylation of rice straw: (A) the crude product straight after the aqueous workup of the reaction mixture; (B) after removing the $\mathrm{CHCl}_{3}$-insolubles.

the acetylation of cellulose reported over the past several years. Most of the operations involved in the preparation of CTA by the two classical methods (entries 1 and 2, Table 2), which have been in use for long are carried out at room temperature. The method using $\mathrm{H}_{2} \mathrm{SO}_{4}$, however, does not usually lead to the desired triacetate, the maximum DS achieved therein being about 2.8. Therefore, for the CTA preparation, it is required to be subjected to another round of reaction in the presence of $\mathrm{HClO}_{4} \cdot{ }^{10}$ The method utilizing $\mathrm{HClO}_{4}$ for the CTA preparation is, however, carried out in the presence of toluene as a cosolvent in addition to the AcOH used. For the successful acetylations, both these methods require quiet lengthy and careful pre-treatments of the substrates (as for the preparations from cotton linters, etc.) as well, owing to which the actual time required for the reaction could be longer than what are shown in the table. The methods employing the ionic liquids ${ }^{14-17}$ as well as molecular iodine, on the other hand, require elevated temperatures $\left(80-100^{\circ} \mathrm{C}\right)$ with one (in the latter case) requiring microwave irradiation ${ }^{21}$ in addition, for high DS of 2.8-3.0 to be obtained. But the iodine-method described by Biswas et al. ${ }^{20,21}$ is indeed exceptionally short and provided the triacetate in a yield of $82 \%$ with isolated cellulose. Likewise, Zhang and co-workers were able to get cellulose acetate of a DS of 2.41 by reaction with $\mathrm{Ac}_{2} \mathrm{O}$ in the presence of an imidazolium-type acidic ionic liquid at $45 \mathrm{~mol} \%$ concentration. ${ }^{35}$ On the other hand Kakuchi et al. reported CTA preparation, again in ionic liquids, but using IPA as the acetyl donor agent with the transesterification having performed at $80{ }^{\circ} \mathrm{C} .{ }^{36}$ The method reported by Liebert and Heinze $^{11}$ employing TBAF/DMSO in the presence of mono-/disodium and potassium hydrogen phosphates at $70{ }^{\circ} \mathrm{C}$ makes use of vinyl acetate as the acyl donor and depending upon the excess of the acyl donor used a DS of up to 2.72 is obtained. ${ }^{11}$ More recent methods reported ${ }^{37-39}$ make use of the classical basic catalyst pyridine and $\mathrm{AcCl}$ in combination with solvents such as $\mathrm{CH}_{2} \mathrm{Cl}_{2}$ or dimethyl acetamide, but only partial acetylations (surface acetylation, consequentially leading to relatively low DSs) have been usually achieved (not listed in Table 2).

While this manuscript was under preparation, a method using $\mathrm{Ac}_{2} \mathrm{O}$ as the acetylating agent in the presence of sulfonated carbon $\left(\mathrm{SO}_{3} \mathrm{H} / \mathrm{PhSO}_{3} \mathrm{H}\right.$-carbon) requiring reactions at $80{ }^{\circ} \mathrm{C}$ for $12 \mathrm{~h}$ was also reported for achieving DSs up to 2.94 (entry 10, Table 2). ${ }^{40}$ In significant advancement to such methods, the methods reported in this investigation yield cellulose triacetate at $40-43{ }^{\circ} \mathrm{C}$ in overnight reactions in a single step (entries 11-17, Table 2). Either neat $\mathrm{AcOH}$ or a mixture of $\mathrm{AcOH}-\mathrm{IPA}$ or $\mathrm{AcOH}-\mathrm{AcCl}$, or alternatively, neat $\mathrm{Ac}_{2} \mathrm{O}$ can be used for the reaction that is catalyzed by MeSA. The cellulose triacetate was invariably obtained in high yields, the best results

Table 2 A comparison of the literature methods for the tri-O-acetylation of cellulose and cellulosic materials with the current methods

\begin{tabular}{|c|c|c|c|c|c|c|c|}
\hline \multirow[b]{2}{*}{ Entry } & \multirow[b]{2}{*}{ Material used } & \multicolumn{4}{|l|}{ Reaction condition } & \multirow[b]{2}{*}{$\%$ yield \& (DS) reported } & \multirow[b]{2}{*}{ Ref. } \\
\hline & & Reagent(s) & Catalyst(s) & Temp $\left({ }^{\circ} \mathrm{C}\right)$ & Time (h) & & \\
\hline 1 & Cotton linters & $\mathrm{Ac}_{2} \mathrm{O}+\mathrm{AcOH}$ & $\mathrm{H}_{2} \mathrm{SO}_{4}$ & $\mathrm{rt}$ & 2 & Not given (2.81) & 10 \\
\hline 2 & Cotton linters & $\mathrm{Ac}_{2} \mathrm{O}+\mathrm{AcOH} / \mathrm{PhMe}$ & $\mathrm{HClO}_{4}$ & $\mathrm{rt}$ & 1 & Not given (triacetate) & \\
\hline 3 & Cellulose & $\mathrm{Ac}_{2} \mathrm{O}$ & AMIMCl & 80 & 8 & Not given (2.49) & 14 \\
\hline 4 & Cellulose & $\mathrm{Ac}_{2} \mathrm{O}$ & {$[\mathrm{ChCl}]\left[\mathrm{ZnCl}_{2}\right]_{2}$} & 90 & 3 & Not given (47\%) & 17 \\
\hline 5 & Cellulose $^{a}$ & $\mathrm{Ac}_{2} \mathrm{O}$ & $\mathrm{I}_{2}$ & 100 & $10 \mathrm{~min}$ & 82 (triacetate) & 20 \\
\hline \multirow[t]{2}{*}{6} & (i) MCC & $\mathrm{Ac}_{2} \mathrm{O}$ & $\mathrm{I}_{2}$ & 100 & 1 & Not given (triacetate) & 19 \\
\hline & (ii) Sisal & $\mathrm{Ac}_{2} \mathrm{O}$ & $\mathrm{I}_{2}$ & 100 & 3 & Not given $(2.8)$ & \\
\hline 7 & Cellulose $^{b}$ & $\mathrm{Ac}_{2} \mathrm{O}$ & $\mathrm{I}_{2}$ & $100^{c}$ & $10 \mathrm{~min}$ & $78(2.8)$ & 21 \\
\hline 8 & Avicel (Fluka) & Vinyl acetate & DMSO/TBAF & 40 & 70 & Not given (1.04-2.72) & 11 \\
\hline 9 & $\operatorname{MCC}^{a}$ & $\mathrm{AcCl}$ & {$\left[\mathrm{C}_{4} \mathrm{mim}\right]^{+} \mathrm{Cl}^{-}$} & 80 & 2 & 86 (triacetate) & 15 \\
\hline 10 & Cellulose & $\mathrm{Ac}_{2} \mathrm{O}$ & $\mathrm{SO}_{3} \mathrm{H} / \mathrm{PhSO}_{3} \mathrm{H}$-carbon & 80 & 12 & $2-2.94$ & 40 \\
\hline 11 & $\mathrm{MCC}^{d}$ & $\mathrm{AcOH}+\mathrm{IPA}$ & MeSA & $40-43$ & 18 & 99 (triacetate) & This work \\
\hline 12 & $\mathrm{MCC}^{d}$ & $\mathrm{AcOH}$ & MeSA & $40-43$ & 18 & 98 (triacetate) & \\
\hline 13 & $\mathrm{MCC}^{d}$ & $\mathrm{AcOH}+\mathrm{AcCl}$ & None additional & $40-43$ & 18 & 90 (triacetate) & \\
\hline 14 & $\mathrm{MCC}^{d}$ & $\mathrm{Ac}_{2} \mathrm{O}$ & MeSA & $40-43$ & 18 & 93 (triacetate) & \\
\hline 15 & Cellulose $^{a}$ & $\mathrm{AcOH}+\mathrm{IPA}$ & MeSA & $40-43$ & 18 & 98 (triacetate) & \\
\hline 16 & Cellulose $^{a}$ & $\mathrm{AcOH}$ & MeSA & $40-43$ & 18 & 60 (triacetate) & \\
\hline 17 & Cellulose $^{a}$ & $\mathrm{AcOH}+\mathrm{AcCl}$ & None additional & $40-43$ & 18 & 55 (triacetate) & \\
\hline
\end{tabular}

${ }^{a}$ Purchased from Aldrich. ${ }^{b}$ Isolated from Caragana korshinskii. ${ }^{c}$ Reaction was done under microwave condition. ${ }^{d}$ Microcrystalline cellulose (MCC) was purchased from SD Fine. 
being obtained in the case of methods using AcOH-MeSA (method B) and IPA-AcOH-MeSA (method A). These latter methods were also therefore performed on straw at $10 \mathrm{~g}$ scale without encountering any practical difficulties or affecting the yield of CTA. Further, it may also be noted that the time taken for the reaction can be reduced considerably in the case of AcOH-IPA reaction if the by-product acetone is removed by distillation which requires the reaction to be carried out at temperatures of $60{ }^{\circ} \mathrm{C}$ or above. When the acetone produced is removed by distillation slowly, the acetylation can be considered to be complete when the collection of acetone (as distillate) stops. The acetone obtained as by-product can thus be collected and put to use elsewhere.

\section{Experimental}

\section{Materials and methods}

Cellulose, AcOH, AcCl and IPA (99\%) were purchased from Sigma-Aldrich, India. MeSA (98\%) was from Alfa-Aesar, John Matthey India. MCC, $\mathrm{Ac}_{2} \mathrm{O}$ (AR grade), $\mathrm{Na}_{2} \mathrm{CO}_{3}$ and $\mathrm{NaHCO}_{3}$ were procured from $\mathrm{S}$. D. Fine-Chem Ltd. Kugelrohr glass oven (B-585) used for drying the samples was from Büchi Labortechnik $\mathrm{GmbH}$. Rice straw was procured directly from a local (Mohali, Punjab) agricultural farm. ${ }^{1} \mathrm{H} /{ }^{13} \mathrm{C}$ NMR spectra were recorded in $\mathrm{CDCl}_{3}$ at $400 / 100 \mathrm{MHz}$ on a Bruker Avance ${ }^{\mathrm{III}}$ spectrometer. The chemical shifts have been reported in ppm using TMS as the internal standard.

\section{Typical procedure for the acetylation of cellulosics}

Cellulose (or other cellulosics such as MCC/cotton linters, $1 \mathrm{~g}$ ) was added to a mixture of: (i) method A: AcOH (10 mL), IPA (10 $\mathrm{mL})$ and MeSA (1 mL) or (ii) method B: AcOH $(20 \mathrm{~mL})$ and MeSA $(1 \mathrm{~mL})$ or (iii) method C: AcOH $(20 \mathrm{~mL})$ and AcCl $(5 \mathrm{~mL})$ in a round bottom flask and the mixture was stirred overnight at 40-43 ${ }^{\circ} \mathrm{C}$. During this period, all the cellulosic material taken initially would have gone into solution and the reaction mixture would have become viscous and jelly. The mixture was then poured in the form of a thin stream into a beaker containing crushed ice and sodium carbonate/sodium bicarbonate while being stirred. The mixture was allowed to stand in the refrigerator for enabling precipitation of the product to complete. The product was then separated at the pump by filtration and after washing with cold water until free of the salts, it was dried in the air followed by drying under vacuum in a Kugelrohr glass oven to constant weight.

\section{Typical procedure for the acetylation of rice straw}

Coarsely pulverized rice straw (please see Fig. 4 above for a photomicrograph, $1 \mathrm{~g}$ ) was added to mixture containing $\mathrm{AcOH}$ $(10 \mathrm{~mL})$, IPA $(10 \mathrm{~mL})$ and MeSA $(1 \mathrm{~mL})$ and was stirred for overnight at $40-43{ }^{\circ} \mathrm{C}$. All of the rice straw taken had gone into solution by this time and the reaction mixture turned viscous and jelly/swollen. It was poured, while being stirred, on to crushed ice containing sodium carbonate/sodium bicarbonate in a beaker. The mixture was left in the refrigerator for the precipitation to complete. It was then separated by filtration at the pump and was dried under reduced pressure. The residual material thus obtained was then taken up in chloroform and was filtered through a fritted glass filter. The insolubles (containing lignin, hemicellulose, silica, etc.) on the filter were washed thoroughly with chloroform. The combined filtrate (clear and light yellowish brown-coloured) was then concentrated to dryness under a vacuum to obtain the rice strawderived CTA as a transparent film $(0.43 \mathrm{~g}, 62 \%$ based on an estimated cellulose content of $40 \%$ in rice straw).

The reactions employing the other methods (AcOH-MeSA/ $\mathrm{AcOH}-\mathrm{AcCl} / \mathrm{Ac}_{2} \mathrm{O}-\mathrm{MeSA}$ ) can also be performed as described above using the respective reagents as required in each case. For the details on the yields obtained, see Table 1.

\section{Characterization of CTA}

The data from FTIR and the ${ }^{1} \mathrm{H}$ and ${ }^{13} \mathrm{C}$ NMR spectral analyses of the synthesized cellulose derivatives were compared with literature data and were found to be in agreement. The powder XRD data (see below) obtained for CTA prepared from rice straw confirmed that the product was not only amorphous but also that it had become free of any silica by dissolution in chloroform and filtration. Elemental analysis of the product confirmed that sulfur was not present in the triacetate prepared.

\section{Conclusions}

This investigation reports three highly efficient, practical and scalable, alternate methods for high yielding (60 to 70\%) production of cellulose triacetate directly from farm crop spare biomass such as rice straw without involving the use of any restricted/toxic chemical ingredients in the process. A typical triacetate preparation implies approximately 30 to $35 \%$ of biomass consumption in acetylation. Since rice straw has about $40 \%$ cellulose, 60 to $87 \%$ acetylation efficiency is thus implied. This is far above the previous reports on rice tissues or their derived cellulose samples. Therefore from a bio refinery perspective, the methods offer advantages of yield and simplicity in the case of acetylation of cellulose (separated from hemicellulose and lignin by biomass fractionation) as an aid to integrated processing of biomass. The method uses a cheap and green organic acid catalyst and provides a proof of principle for a way forward to valorise a zero-use and problematic farm residues like rice straw.

\section{Acknowledgements}

A. S. sincerely thanks CIAB, Mohali \& the Department of Biotechnology (DBT), New Delhi for financial assistance and S. K. G. sincerely thanks NIPER, S. A. S. Nagar and the Ministry of Chemicals and Fertilizers, Govt. of India for permission to pursue Ph.D. under its staff development scheme.

\section{Notes and references}

1 Y. Habibi, L. A. Lucia and O. J. Rojas, Chem. Rev., 2010, 110, 3479-3500. 
2 R. Rinaldi and F. Schuth, Energy Environ. Sci., 2009, 2, 610626.

3 R. P. Swatloski, S. K. Spear, J. D. Holbrey and R. D. Rogers, J. Am. Chem. Soc., 2002, 124, 4974-4975.

4 I. Cumpstey, ISRN Org. Chem., 2013, 1-27.

5 D. G. Barkalow, R. M. Rowell and R. A. Young, J. Appl. Polym. Sci., 1989, 37, 1009-1018.

6 I. A. Wolff, D. W. Olds and G. E. Hilbert, J. Am. Chem. Soc., 1951, 73, 346-349.

7 S. Richardson and L. Gorton, Anal. Chim. Acta, 2003, 497, 2765.

8 R. L. Shogren, Carbohydr. Polym., 2003, 52, 319-326.

9 K. J. Edgar, C. M. Buchanan, J. S. Debenham, P. A. Rundquist, B. D. Seiler, M. C. Shelton and D. Tindall, Prog. Polym. Sci., 2001, 26, 1605-1688.

10 L. J. Tanghe, L. B. Genung, and J. W. Mensch, in Methods in Carbohydrate Chemistry, ed. R. L. Whistler and M. L. Wolfrom, Academic Press, New York, NY, USA, 1963, vol. III, Cellulose, pp. 193-212.

11 T. F. Liebert and T. J. Heinze, Biomacromolecules, 2001, 2, 1124-1132.

12 T. Welton, Chem. Rev., 1999, 99, 2071-2084.

13 H. L. Ngo, K. LeCompte, L. Hargens and A. B. McEwen, Thermochim. Acta, 2000, 357, 97-102.

$14 \mathrm{~J}$. Wu, J. Zhang, H. Zhang, J. He, Q. Ren and M. Guo, Biomacromolecules, 2004, 5, 266-268.

15 S. Barthel and T. Heinze, Green Chem., 2006, 8, 301-306.

16 A. Schenzel, A. Hufendiek, C. Barner-Kowollik and M. A. R. Meier, Green Chem., 2014, 16, 3266-3271.

17 A. P. Abbott, T. J. Bell, S. Handa and B. Stoddart, Green Chem., 2005, 7, 705-707.

18 K. P. R. Kartha and R. A. Field, Tetrahedron, 1997, 53, 1175311766.

19 M. P. Paula, T. M. Lacerda and E. Frollini, eXPRESS Polym. Lett., 2008, 2, 423-428.

20 A. Biswas, R. L. Shogren and J. L. Willett, Biomacromolecules, 2005, 6, 1843-1845.
21 J. Li, L. P. Zhang, F. Peng, J. Bian, T. Q. Yuan, F. Xu and R. C. Sun, Molecules, 2009, 14, 3551-3566.

22 A. Biswas, B. C. Saha, J. W. Lawton, R. L. Shogren and J. L. Willett, Carbohydr. Polym., 2006, 64, 134-137.

23 S. K. Giri and K. P. R. Kartha, RSC Adv., 2015, 5, 11687-11696.

24 D. C. Hull, O. Ridge and A. H. Agett, US Pat., 2422 016, 1947.

25 H. J. Hagemeyer and D. C. Hull, Ind. Eng. Chem., 1949, 41, 2920-2924.

26 E. A. Jeffery and D. P. N. Satchell, J. Chem. Soc., 1962, 18761887.

27 E. A. Jeffery and D. P. N Satchell, J. Chem. Soc., 1962, 18871894.

28 D. P. N. Satchell, J. Chem. Soc., 1962, 1894-1898.

29 D. P. N. Satchell, J. Chem. Soc., 1962, 1899-1905.

30 E. A. Jeffery and D. P. N. Satchell, J. Chem. Soc., 1962, 19131917.

31 R. Pelagalli, I. Chiarotto, M. Feroci and S. Vecchio, Green Chem., 2012, 14, 2251-2255.

32 C. E. Bronnimann, R. C. Zeigler and G. E. Maciel, J. Am. Chem. Soc., 1998, 110, 2023-2026.

33 H. E. L. Rassy, P. Buisson, B. Bouali, A. Perrard and A. C. Pierre, Langmuir, 2003, 19, 358-363.

34 H. E. l. Rassy and A. C. Pierre, J. Non-Cryst. Solids, 2005, 351, 1603-1610.

35 X. Zhang, W. Zhang, D. Tian, Z. Zhou and C. Lu, RSC Adv., 2013, 3, 7722-7725.

36 R. Kakuchi, M. Yamaguchi, T. Endo, Y. Shibata, K. Ninomiya, T. Ikai, K. Maeda and K. Takahashi, RSC Adv., 2015, 5, 72071-72074.

37 T. Mukherjee, M. Sani, N. Kao, R. K. Gupta, N. Quazi and S. Bhattacharya, Chem. Eng. Sci., 2013, 101, 655-662.

38 F. Hu, N. Lin, P. R. Chang and J. Huang, Carbohydr. Polym., 2015, 129, 208-215.

39 S. A. Ganie, A. Ali and N. Mazumdar, Carbohydr. Polym., 2015, 129, 224-231.

40 L. J. Konwar, P. Mäki-Arvela, A. J. Thakur, N. Kumar and J. Mikkola, RSC Adv., 2016, 6, 8829-8837. 